\title{
Force Calibration with Build Up Systems
}

Thomas Kleckers, Dr. Andre Schaefer

Hottinger Baldwin Messtechnik GmbH, Im Tiefen See 45; 64293 Darmstadt, Germany

Author: Thomas.kleckers@HBM.com

\begin{abstract}
The idea behind a build-up system is to use force transducer with a lower capacity in a mechanical parallel arrangement to increase the capacity of the complete system. Three or more individual sensors are completed to a build-up system. The aim of those systems is to calculate the behavior of the complete system from the calibration of the individual sensors.
\end{abstract}

Keywords / Mots Clés: Build up systems, force reference transducer, parallel circuit of strain gauge based sensors

\section{Calibration of force transducers, application of build-up systems}

Two different methods to calibrate force transducers are common.

Especially for lower capacities so called dead load machines are used. With those machines weights are loaded to the sensor to apply a certain reference force. The largest system in Germany is owned by the national metrological institute the "Physikalisch Technische Bundesanstalt" (PTB). This system can load force transducers with a force of $2 \mathrm{MN}$.

It can be seen that the calibration of force transducers with higher capacities is very difficult with dead load machines as the required masses are enormous. Therefore hydraulic systems take place in this field. A very common method is to use hydraulic cylinders and a reference sensor for calibration. With those systems the sensor to be calibrated is in a serial arrangement with the reference load cell. The signal of the reference sensor is compared with the output of the transducer under test.

The upcoming question with those systems is how to calibrate the reference sensor in case of forces in the Mega - Newton range. The reference sensors of the calibration system have also to be calibrated, but dead load machines are not available in this range. There is the need to find a way to use the result of lower capacity calibration machines for the calibration of high capacity force transducer. As the demand for high capacity force transducers is visible on the marked this question becomes more and more important.

Build-up system could help to overcome this problem as the individual sensors can be calibrated precisely on low capacity calibration machines. After having the calibration results of the single sensor the system can be arranged to work in parallel and the output at the higher forces can be predicted. 
The important assumption is that the behavior of the complete system can be calculated out of the calibrations of the individual sensors.

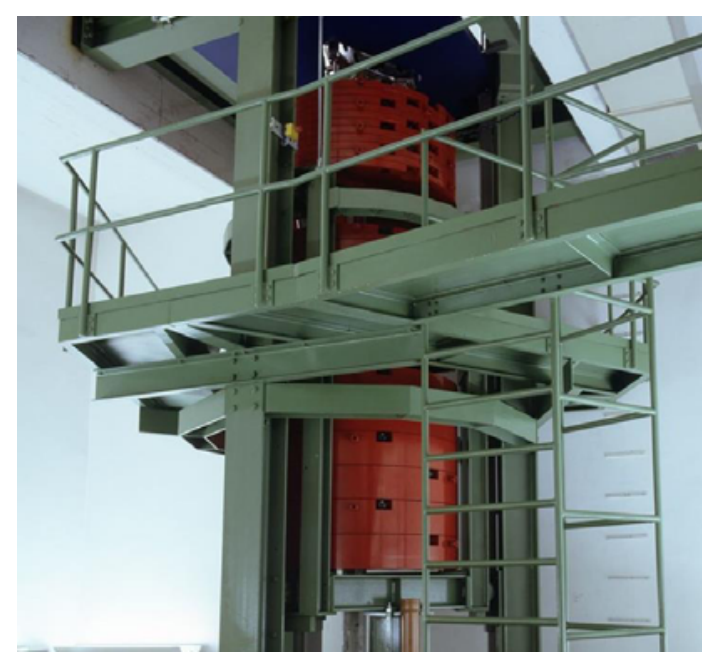

Fig. 1: $240 \mathrm{KN}$ dead load calibration machine from HBM. The sensors can be loaded in tension and compression with the calibrated weights

\section{Mechanical design of build-up systems}

A build-up - system consists of the sensors and massive plates for load introduction. The stiffness of the base plate and the plate arranged above the sensors is of highest importance. (Fig. 2)

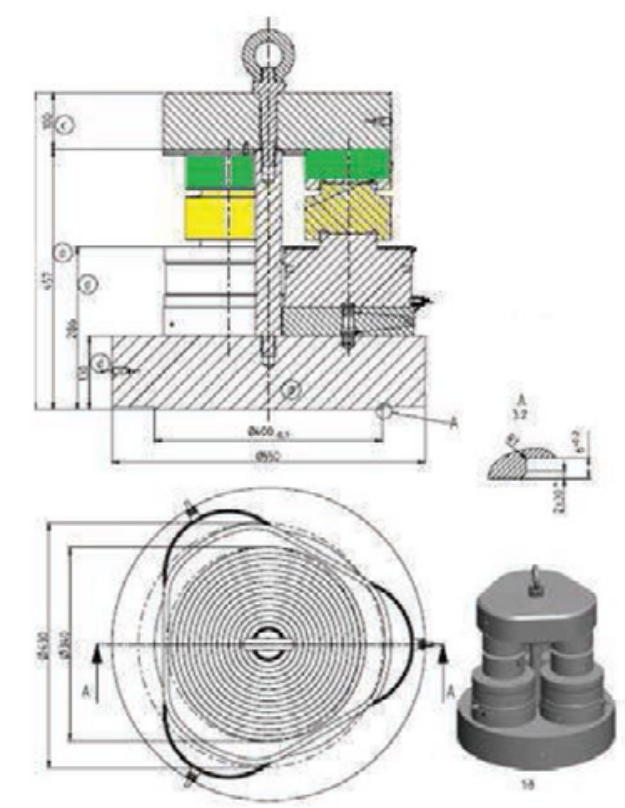

Fig. 2: A modern Build - up system consisting three sensors with a capacity of $2 \mathrm{MN}$ each. (HBM BU18/6MN) 
The reason for this is that the plates will deform under load. This means that the force sensors will not be any longer in precise line with the force vector- a measurement uncertainty will occur.

A pendulum mount (yellow) and a thrust piece (green) help to minimize the effect of upcoming torque and bending. This can be the case due to effects of the calibration machine, but also due to deformation of the load introduction plates.

Furthermore the parallelism of the upper and lower base plates are important to ensure that the load is distributed in similar portions to the singlel sensors. This is ensured by a grinding process at the end of the production of a build - up system. Every arrangement has the same height after this. Furthermore the sensor, the thrust piece and the pendulum are marked clearly for each unit so that the system can be rearranged in a way so that all components are always placed at the same position in the same orientation.

The stiffness and therefore the deflection of the three sensors should show a low tolerance only.

\section{Electrical connection of the Build - up systems}

In most cases each sensor is connected to an individual amplifier channel. The calculation of the force can be easily done by adding the individual forces. Nevertheless the sensors can be connected in parallel. Therefore the so called $\mathrm{R} / \mathrm{C}$ adjustment is important which means that the relation between output signal and output resistance must be constant. This adjustment is done under normal conditions the fine adjustment can be achieved by using special connection boxes which allow a precise setup.

\section{Some Results}

A build up system with a capacity of $6 \mathrm{MN}$ in total was calibrated in two different ways. One calibration has been performed with the complete system. Secondly each of the three sensors was subject of a calibration in accordance to the ISO376 standard. The results of the individual sensors are given in tab 1.

The capacity of parallel connected load cells must be added while the output remains the same for the theoretical output signals. [1]

The results in table 2 show the calculation based on the results of the single sensors and the theoretical parallel arrangement and the comparison with the system calibration of the complete build-up system. As the calibration steps are different a linear interpolation was used to evaluate the theoretical output. (Exception: $3000 \mathrm{kN}$ ). 
Tab1: Output signal of the three individual sensors. Numbers show the mean values in rotated mounting positions rotated mounting positions

\begin{tabular}{|r|r|r|r|}
\hline Force [kN] & $\begin{array}{c}\text { Output } \\
\text { transducer 1 } \\
{[\mathrm{mV} / \mathbf{V}]}\end{array}$ & $\begin{array}{c}\text { Output } \\
\text { transducer 1 } \\
{[\mathrm{mV} / \mathrm{V}]}\end{array}$ & $\begin{array}{c}\text { Output } \\
\text { transducer 1 } \\
{[\mathrm{mV} / \mathbf{V}]}\end{array}$ \\
\hline 200 & 0,263759 & 0,263733 & 0,263963 \\
\hline 400 & 0,527678 & 0,527552 & 0,527965 \\
\hline 600 & 0,791691 & 0,791474 & 0,792066 \\
\hline 800 & 1,055725 & 1,055414 & 1,05621 \\
\hline 1000 & 1,319869 & 1,319468 & 1,320363 \\
\hline 1200 & 1,584015 & 1,583559 & 1,584596 \\
\hline 1600 & 2,112325 & 2,111751 & 2,112939 \\
\hline 2000 & 2,640616 & 2,639936 & 2,641185 \\
\hline
\end{tabular}

Tab2: Comparison of theoretical calculated values with real calibration of the complete $6 \mathrm{MN}$ system. Base of the theoretical values are the individual calibration of the load cells. Italic numbers are based on a interpolation as the calibration steps do not match.

\begin{tabular}{|c|l|l|l|}
\hline Force $[\mathbf{k N}]$ & $\begin{array}{l}\text { Theoretical output of } \\
\text { the system [mV/V] }\end{array}$ & $\begin{array}{l}\text { Result of the system } \\
\text { calibration [mV/V] }\end{array}$ & Deviation in \% \\
\hline 600 & 0,263818333 & 0,2638466 & $-0,01 \%$ \\
\hline 1200 & 0,527731667 & 0,5277306 & $0,00 \%$ \\
\hline 1800 & 0,791743667 & 0,7917114 & $0,00 \%$ \\
\hline 2400 & 1,055783 & 1,0558386 & $-0,01 \%$ \\
\hline 3000 & 1,3199 & 1,319798 & $0,01 \%$ \\
\hline 3600 & 1,584056667 & 1,5840556 & $0,00 \%$ \\
\hline 4800 & 2,112338333 & 2,1122652 & $0,00 \%$ \\
\hline 6000 & 2,640579 & -- & -- \\
\hline
\end{tabular}

Reasons for the deviation are given by four points mainly:

- The calibration machine used has an uncertainty of $0.02 \%$ and is therefore no negligible error

- The stiffness of the individual setup consisting a sensor, the thrust piece and the pendulum mount is not the same for all three arrangements so that the load is not divided with exactly the same amount on each sensor 
- The adjustment of the output resistance and the rated output is done with a certain tolerance

- The upper and lower plate is not perfectly stiff, so that bending occurs. As a result the load cells are no more exactly parallel to the force vector.

\section{Conclusion}

Build us systems can increase the possibility to calibrate sensors in the $\mathrm{MN}$ - Range in the future. Current systems show a not acceptable deviation from the theoretical output compared with the measured system output. Further research is necessary to develop proper correction methods.

The systems are extreme reliable and do reach the accuracy class 00 according the ISO376 standard.

\section{Reference}

[1] Jürgen Paetow, Parallelschaltung von DMS - Wägen und Dosieren A usgabe $6 / 1987$ 